\title{
Selection of the operation for duodenal ulcer
}

The selection of the operation for duodenal ulcer has been a subject of discussion and argument for forty years. In the thirties the problem was whether pyloroplasty or gastrojejunostomy was the superior operation. Later came the problem of the relative merits of partial gastrectomy and gastrojejunostomy. The revival of vagotomy and drainage brought an alternative to partial gastrectomy, and vagotomy seems to have won favour as judged by its greater use. Over the years each operation has then been found to have disadvantages which have led to its rejection for a new procedure. Now we are discovering the disadvantages of truncal vagotomy, and so some have reverted to partial gastrectomy or, more logically, have modified vagotomy by making a selective or gastric vagotomy only.

These various mutilations of the stomach have been a great stimulus to the study of physiology, and nowadays when surgery has become more of a science and perhaps less of an art, we like to feel that we plan our operative procedures on sound physiological principles. We can now fairly accurately measure the maximal acid secretion of the stomach and from this estimate the parietal cell population. From this has arisen the concept of selecting a particular operation to suit a particular patient according to his secretory capacity. This concept has never been a very reliable guide, for we have no idea how often, if ever, in a particular patient the stomach secretes at or near its maximal capacity. We cannot measure our patients' day-by-day stresses, and so the patient with a relatively low maximal acid output, who is chosen for a simple gastrojejunostomy may thereafter secrete maximally and develop a stomal ulcer. Again, in patients with high maximal acid output it is often recommended that vagotomy and a partial gastrectomy be carried out. Such a procedure almost eliminates the chance of getting a stomal ulcer, but it increases the operative risk and chances of side effects, in most cases quite unnecessarily, because either of these operations by itself will give a 96 to $98 \%$ ulcer cure rate. Perhaps the nearest we can come to selection on the basis of the maximal acid secretion is to state that if a patient with a relatively inactive but grossly scarred duodenal ulcer secretes under $30 \mathrm{~m}$-equiv of $\mathrm{HCl}$ in the post-histamine hour, then it is reasonably safe to employ a simple gastrojejunostomy. ${ }^{1}$

At 'follow-ups' of one to three years nearly all operations for duodenal ulcer have a high proportion of good results, except perhaps simple vagotomy, which is marred by gastric retention in $60 \%$ of the cases. Only after five years or more can one assess the value of an operation. Therefore, no matter how well designed a new operation 
may be, it remains an experiment until it has stood the test of an unbiased long 'follow-up'. A long 'follow-up', minimally five years, is especially important to assess the beneficial effects of an ulcer operation. Because of the natural history of ulcer, with its relapses and remissions, any operation involving a stay in hospital and a convalescence will produce a good remission of ulcer pain in nearly all cases and the remission may last for many months or even for three to four years. This need for late assessment does not apply to the surgery of ulcer bleeding or perforation, where the survival of the patient is the criterion of success, nor does it apply when the intent is to indicate either a high mortality or a high incidence of 'side effects' following an operation.

Partial gastrectomy is an operation of which we now know the best and the worst. On the whole it has given considerable comfort to many thousands of ulcer sufferers with a low recurrent ulcer rate and restoring a reasonable ability to work and enjoy life. Recurrent ulceration occurs in about $2 \%$ and can always be corrected, thanks to vagotomy. Bilious vomiting is present in a small number of cases and can and should always be corrected. ${ }^{2}$ Early dumping, fullness, or mild 'hypoglycaemia-like' attacks may vitiate against complete satisfaction in a small percentage of cases and occasionally mar the early years after operation. The results of gastrectomy in many clinics have shown too high an incidence of side effects because of the emphasis on 'subtotal' gastrectomy for duodenal ulcer and overpreoccupation with the possibility of stomal ulcer. An antrectomy alone reduces the maximum acid response by $65 \%^{3}$ and it is probably never necessary to do more than a $60 \%$ resection for duodenal ulcer. In these high resections many surgeons divide the main trunk of the left gastric artery, which usually means division of the coeliac branch of the posterior vagus nerve, and so postvagotomy symptoms may be added to the patient's troubles.

Varying figures are given for recurrent ulceration after truncal vagotomy, the higher figures probably representing a lack of familiarity with the anatomy of the vagus. It is notable that recurrent ulceration rates are higher when a follow up of the results of the work of the whole surgical staff of a hospital, some of whom may have some other main interest, is given, than when the results of an interested gastroenterological surgeon are reported. The 'side effects' which may follow truncal vagotomy are now well known: episodic diarrhoea in 1 to $5 \%$ of the cases, hypoglycaemia, dumping symptoms, and bilious regurgitation in a variable number, according to the standards of assessment employed. The diarrhoea tends to improve slightly over a long period though there were still five with severe episodic diarrhoea out of 143 of the reviewer's cases of truncal vagotomy studied 10 to 20 years after operation. ${ }^{4}$ Episodic diarrhoea is most resistant to treatment though the use of a reversed ileal loop seems to be a promising method of treatment. The dumping and hypoglycaemia are at least in part associated with the drainage procedure. Without 
any form of drainage at all truncal vagotomy is satisfactory in some $40 \%$ of the cases. It is for the $60 \%$ who would suffer gross delay in emptying after vagotomy that we now employ a routine drainage procedure. A pyloroplasty once made cannot be repaired, and so there is something to be said for utilizing a gastrojejunostomy, which can be eliminated. Conversion from gastrojejunostomy to pyloroplasty brings only slight benefits to patients with diarrhoea or dumping, and so in a severe case it might be better merely to dismantle and repair the gastrojejunostomy, leaving the pylorus intact provided that there is no gross duodenal stenosis.

The reviewer's opinion, and that of the physicians and registrars who have studied the cases, is that the results of total vagotomy and drainage, though in the majority of cases highly gratifying, are on the whole marginally less satisfactory than those of partial gastrectomy. The greatest advantage of truncal vagotomy is its lower mortality up and down the country.

As far back as 1948 it was agreed that the loss of the parasympathetic nerve fibres to the pancreas, small and proximal large intestine, to the liver and bile ducts was an undesirable feature of truncal vagotomy. However, most surgeons believed that we should first discover the effects of truncal vagotomy before introducing variations on the operation, particularly as in the main it was a reasonably satisfactory procedure. However, we now know the five to 10 year results and the 15 to 20 year follow-up figures are coming in, and so the time has come to evaluate selective gastric vagotomy.

A great many selective vagotomy operations have now been carried out but few really long-term studies are available. In most series the period of follow up is inadequate to speak with any certainty of recurrent ulceration, but it will probably prove to be about the same percentage as with truncal vagotomy. However, preliminary check follow ups have shown a notable reduction in the incidence of episodic diarrhoea. Fullness after meals and hypoglycaemia-like attacks still occur despite selective vagotomy and these must at least in part be attributable to damage to or bypassing of the pylorus.

It is possible, as Burge ${ }^{5}$ believes, that the pyloric innervation is less disturbed by selective vagotomy than by truncal vagotomy and that no drainage procedure is necessary with selective vagotomy, unless there is coincident duodenal stenosis. If this can be confirmed then it would be a most useful step forward.

NORMAN C. TANNER

\section{REFERENCES}

\footnotetext{
${ }^{1}$ Small, W. P. (1964). Recurrence of duodenal ulcer after gastro-enterostomy. J. roy. Coll. Surg. Edinb. 9, $141-145$.

${ }^{2}$ Tanner, N. C. (1966). Disabilities which may follow the Peptic Ulcer Operation. Presidential Address. Proc. roy. Soc. Med., 59, 362-368.

${ }^{3}$ Gillespie, I. E., Clark, D. H., Kay, A. W., and Tankel, H. I. (1960). Effect of antrectomy, vagotomy with gastrojejunostomy, and antrectomy with vagotomy on the spontaneous and maximal gastric output in man. Gastroenterology, 38, 361-367.

'Wastell, C. (1969). Personal communication.

${ }^{5}$ Burge, H. W. (1969). Personal communication.
} 